\title{
El análisis de tareas para el desarrollo de competencias argumentativas en geometría ${ }^{1}$
}

\section{RESUMEN}

Este artículo presenta resultados de investigación en la relación desarrollo de lenguaje y desarrollo de conocimiento matemático, desde una perspectiva argumentativa. La propuesta parte de la hipótesis fundamental de que el desarrollo de un argumento matemático depende de una relación básica entre la lengua natural como sistema semiótico que privilegia la argumentación y los razonamientos en general y los sistemas semióticos como el algebraico y el figural que se privilegian en los razonamientos matemáticos en la elaboración de conceptos matemáticos como los geométricos. Desde este punto de vista, en el presente estudio está el interés por comprender, describir, analizar y cualificar los procesos de aula, tanto en la dimensión de la enseñanza como en la del aprendizaje, en los campos particulares del lenguaje y las matemáticas. Así,

Este artículo es parte de la tesis doctoral de la autora, titulada "Dimensión cognitiva y comunicativa de la argumentación en matemáticas", sustentada y aprobada en diciembre de 2005. Universidad del Valle. Además, gran parte de sus presupuestos teóricos y metodológicos son compartidos con los de la tesis de la profesora Olga Lucía León titulada "Experiencia figural y procesos semánticos para la argumentación en geometría".

2 Doctora en Educación con énfasis en Lenguaje de la Universidad del Valle, miembro del Grupo de Investigación Interdisciplinaria en Pedagogía del Lenguaje y las Matemáticas y Profesora de planta de la Universidad Distrital Francisco José de Caldas de Bogotá. 
en este documento se presenta, como resultado de la investigación, una estructura de análisis de tarea de uno de los talleres del diseño didáctico global. Se presenta tanto el análisis a prioricomo el a posteriori, que se realiza desde el modelo de la ingeniería didáctica (Guy Brousseau, 1998, 2004 y Régine Douady, 1984, entre otros). El diseño de tareas se encaminó al desarrollo de competencias argumentativas en geometría, en un grupo de estudiantes de $10^{\circ}$ grado de educación media. Se realiza una estructuración específica para el análisis de una secuencia de actividades orientadas al desarrollo de procesos de razonamiento (como el argumentativo) y su relación con los procesos de representación (como los semióticos), en la formulación de una relación geométrica: la relación pitagórica ${ }^{3}$.

\section{Palabras clave:}

Didáctica del lenguaje, análisis de tareas, diseño didáctico, comunicación en el aula y competencia argumentativa en geometría.

\section{ABSTRACT}

This article presents results of a research in the relation language development and development of mathematical knowledge, since an argumentative perspective. The proposal splits of the fundamental hypothesis that the development of a mathematical argument depends on a basic relation among the natural language as semiotic system that privileges the argument and the reasoning in general, and the semiotic systems like the algebraic one and the figural one, privileged in the mathematical reasoning in the elaboration of mathematical concepts such as the geometric concept. From that point of view, in the present study exists the interest to understand, to describe, to analyze and to qualify the processes in the classroom, both in the dimension of the teaching act and in the learning one, in the private fields of the language and the math. Thus, in this document is presented, as a consequence of the investigation, an structure of task analysis from one of the workshops of the global didactic design. Both the analysis are presented a priori and a posteriori, made from the model of the didactic engineering (Guy Brousseau, 1998, 2004, and Régine Douady, 1984, among others). The design of tasks was directed to the development of argumentative competences in geometry, in a group of students of $10^{\circ}$ degree of secondary education. A specific structuring for the analysis of a sequence of activities oriented is carried out to the reasoning processes development (as the argumentative one) and its relation with the processes of representation (like the semiotics process), in the formulation of a geometric relation: the Pythagoream relation. ${ }^{6}$

\section{Keywords:}

Language teaching, tasks analysis, didactic design, communication in the classroom, and, argumentative competence in geometry.

3 El análisis de la Relación Pitagórica como contenido matemático es desarrollado por la profesora Olga Lucía León (2005), en el trabajo complementario a este estudio. 


\section{INTRODUCCIÓN}

Esta investigación interdisciplinar de tipo pedagógico y didáctico, se realiza en el campo de la enseñaza y del aprendizaje del lenguaje y de las matemáticas. Su interés fundamental es el desarrollo de competencias argumentativas en geometría; para ello pretende: 1) Identificar factores relacionados con la enseñanza y con el aprendizaje de la argumentación èn matemáticas. 2) Establecer relaciones entre las dimensiones comunicativa-argumentativa y cognitiva-geométrica. De ahí que la pregunta general que nos formulamos es por el tipo de factores que están en la génesis de la elaboración de los argumentos, que enmarcan el posicionamiento argumentativo de los estudiantes en la solución de problemas matemáticos.

Un supuesto básico que orientó el estudio es que el desarrollo de la argumentación depende de una estrecha relación entre el lenguaje como discurso, los procesos de razonamiento y los sistemas semióticos propios del campo en el que se argumenta. Desde este punto de vista, en el presente estudio está el interés por comprender, describir, analizar y cualificar los procesos de aula, tanto en la dimensión de la enseñanza como en la del aprendizaje, en los campos particulares del lenguaje y las matemáticas. Para ello se realiza, desde el modelo de la ingeniería didáctica (Guy Brousseaú, 1998, 2004 y Régine Douady, 1984, entre otros) un trabajo de análisis de tareas tanto a prioricomo a posteriori, encaminadas al desarrollo de competencias argumentativas en geometría, en un grupo de estudiantes de $10^{\circ}$ grado de educación media. Se realiza una estructuración específica para el análisis de una secuencia de actividades didácticas orientadas al desarrollo de procesos de razonamiento (como el argumentativo) y su relación con los procesos de representación (como los semióticos), en la formulación de una relación geométrica: la relación pitagórica.

La reflexión que hemos realizado alrededor del desarrollo de competencias argumentativas en matemáticas nos lleva a estudiar factores de tipo cognitivo y de tipo comunicativo que inciden en el desarrollo de procesos argumentativos efectivos, durante los procesos de negociación de sentido y de significados matémáticos que realizan los estudiantes. En esta perspectiva, nos interesa establecer aspectos fundamentales presentes en el desarrollo de la argumentación en matemáticas; en particular, identificar factores de tipo cognitivo, comunicativo, epistemológico y didáctico que inciden en la producción del discurso argumentativo en geometría euclidiana. Con esta base se pretende formular estrategias didácticas para el desarrollo de procesos argumentativos en matemáticas.

\section{SUPUESTOS TEÓRICOS DE BASE}

Esta propuesta tiene como base los siguientes supuestos y consideraciones:

a. Un factor fundamental de los procesos de elaboración de conocimiento y de comunicación en el aula, lo constituye el empleo de la argumentación (Martínez, 2001, 2004b, 2005; Calderón y León, 2001,2003) en particular, en situaciones de legitimación de conocimientos y procedimientos matemáticos (Duval, 2001).

b. La práctica argumentativa manifiesta niveles de elaboración de conocimientos y la presencia de normas de interacción social. Además implica la confrontación, la 
modificación y la estructuración de saberes particulares (Perelman y Olbrecht-Tyteca, 1989; Duval, 1999, 2001; Van Eemeren y Grootendorst, 2002; Calderón y León, 2001, 2003, 2005).

c. La práctica argumentativa y la práctica validativa son dos acciones básicas para el desempeño matemático de un estudiante (Duval, 1999, 2001).

d. El desarrollo de competencia argumentativa en geometría en el aula exige la estructuración de situaciones didácticas y, por ello, la aplicación de una teoría didáctica (Marilier, Robert y Tenaud, 1987; Bloch, 2002; Yves Chevallard, 1985, 1986; Calderón y León, 20011.

e. La reflexión sobre el aprendizaje de una relación matemática, en el espacio escolar implica, como aspecto fundamental, el análisis del papel de las actividades propuestas en el aula para desarrollo de un tipo de conocimiento, en relación con el propósito y con el moménto de su aprendizaje. Para ello, es importante considerar un supuesto inscrito en la teoría del constructivismo social (Vigotsky, 1982) como punto de partida: los conocimientos previos o el grado de acercamiento y de experiencia anterior que los estudiantes tengan con el conocimiento en cuestión, determinan procesos de desarrollo.

f. Un análisis del lugar de la argumentación en los currículos colombianos muestra que, al menos para las propuestas curriculares de 1970 en adelante, la inclusión del sentido de "habilidad" contribuyó a considerar la inclusión, en el diseño curricular, de las técnicas deliberativas como recurso metodólógico, aunque no como procedimiento discursivo para la construcción de situaciones argumentativas. En este sentido, el componente argumentativo no llega a ser explícito para el profesor, generando quizás, un tratamiento más temático (descriptivo y ejemplificador) de factores metodológicos y textuales que, asumidos desde otra óptica, propiciarían el desarrollo de la argumentación. Posteriormente, y desde la década del 90 , se da lo que hemos denominado "el auge la argumentación en la escuela" (ver Calderón 2005), en el que se plantea una inclusión tanto teórica como metodológica de la argumentación en los distintos niveles de escolaridad (Ver Lineamientos Curriculares para la Lengua Castellana, 1998, Estándares Curriculares para la Lengua Castellana, 2002. 2003). No obstante, y a partir de un análisis minucioso del tratamiento que se da a la argumentación en estos documentos, es un hecho que ella toma un lugar importante en tanto tema de la educación en básica y media y que se genera una cultura léxica que habla de la argumentación en el ámbito escolar; sin embargo, los planteamientos teóricos y las propuestas de tratamiento de la argumentación en el aula requerirían precisiones tanto de tipo teórico como metodológico, que propicien prácticas pedagógicas más efectivas para su desarrollo (Calderón, 2005).

\section{PROPUESTA DE ANÁLISIS DE TAREAS}

Como se mencionó anteriormente, en el marco de la ingeniería didáctica se realiza una estructura de análisis de tareas en dos momentos: a prioriy a posteriori. El análisis a priori pretende fundamentalmente adecuar el diseño didáctico a condiciones curriculares e identificar unidades significantes en los diseños de actividades. Este análisis se desa- 
rrolla según variables didácticas macroestructurales como el tipo de género discursivo en el que se inscriben las tareas, la situación de comunicación y el tipo de interacción; y según variables microestructurales como las relaciones matemáticas identificadas, las formas de aprehensión del registro figural y las formas de expansión discursiva para la formulación de un enunciado matemático. Asimismo, la noción de tarea que se construye desde este modelo de investigación, se constituye como un sistema propuesto para el desarrollo de los aprendizajes de los estudiantes, que se articula en los niveles macro (curricular y didáctico) y micro (de la interacción con el conocimiento y con los interlocutores del aula), niveles que se han de tener en cuenta en los respectivos análisis.

Posteriormente, como fundamento de esta metodología de investigación, se da la confrontación entre los análisis a priorisobre los diseños de actividades de aula y los análisis a posteriorisobre los corpus que se producen en la implementación de las tareas. Esta acción se constituye en la forma básica de validación de las hipótesis formuladas en la investigación. A continuación se presentan aspectos generales de la propuesta de los dos tipos de análisis, como un aporte a la configuración de la metodología de análisis de tareas para el desarrollo de competencias argumentativas en geometría.

\subsection{El análisis a priori de la tarea o la configuración de un contexto de aprendizaje}

El análisis a priori de las tareas se estructura en dos niveles de análisis: macroestructural y microestructural. El primero define variables relacionadas con el contexto global de la tarea; en este caso del género pedagógico y sus variables macroestructurales asociadas. Así pues, este nivel constituye el contexto general para el desarrollo de la propuesta didáctica. Su análisis permite observar las implicaciones del trabajo didáctico en el contexto escolar. A continuación se visualizan las categorías macro que estructuran la tarea y que indican aspectos determinantes en las decisiones didácticas.

\section{Variables relacionadas con el género pedagógico}

\begin{tabular}{|l|l|}
\hline Contexto escolar & El diseño didáctico: experiencia de enseñanza y aprendizaje. \\
\hline $\begin{array}{l}\text { Modo de organización } \\
\text { de la interacción }\end{array}$ & $\begin{array}{l}\text { Taller: Para propiciar procesos cognitivos y comunicativos en } \\
\text { geometría }\end{array}$ \\
\hline $\begin{array}{l}\text { Situación de } \\
\text { comunicación previa }\end{array}$ & $\begin{array}{l}\text { Didáctica de formulación: determinada por la intencionalidad, } \\
\text { el contenido y la estructura de interacción. }\end{array}$ \\
\hline
\end{tabular}

El primer análisis que se realiza es el relacionado con el contexto en el que se produce la tarea. Dado que se trata del contexto escolar, la tarea se sitúa, desde el nivel macro, en el género discursivo pedagógico y, más específicamente en la relación enseñanzaaprendizaje. Esto se refleja en el diseño didáctico elaborado para toda la secuencia de talleres. La estructura del diseño didáctico se determina por las relaciones dadas por los requerimientos comunicativo, epistemológico, cognitivo y sociocultural del aula (Calderón y León, 2001), como se manifiesta en el tipo de opciones tomadas: 


\begin{tabular}{|c|c|}
\hline $\begin{array}{l}\text { REQUERIMIENTO } \\
\text { DIDÁCTICO }\end{array}$ & OPCIONES TOMADAS \\
\hline $\begin{array}{l}\text { Desde lo } \\
\text { comunicativo }\end{array}$ & $\begin{array}{l}\text { Un tipo de interacción comunicativa que propicia la elaboración con- } \\
\text { tinua del sentido puesto en cuestión y de sus procesos implicados. } \\
\text { Esta es la interacción argumentativa como el espacio comunicativo } \\
\text { que exige: i) el reconocimiento de un tema polémico, en tan- } \\
\text { to que en el aula se están elabórando múltiples sentidos para la re- } \\
\text { lación pitagórica; ii) el reconocimiento de una situación argumen- } \\
\text { tativa, en tanto está la necesidad de justificar el sentido elaborado } \\
\text { ante un auditorio (el profesor y los compañéros); iii) la asunción de } \\
\text { una actitud argumentativa que implica: estar dispuesto a estruc- } \\
\text { turar posiciones individuales en torno al sentido, realizar tramas } \\
\text { argumentativas que manifiesten la posición individual, prever a un } \\
\text { auditorio y producir un discurso argumentativo para convencer o } \\
\text { persuadir a ese auditorio, prever y realizar una actividad de valoración } \\
\text { de argumentos contrarios y estar dispuesto a consensuar. }\end{array}$ \\
\hline $\begin{array}{l}\text { Desde lo } \\
\text { epistemológico }\end{array}$ & $\begin{array}{l}\text { La relación pitagórica como una relación geométrica. Se optó } \\
\text { por la trama argumentativa fundamentada en la relación ángulo rec- } \\
\text { to-teoría de las paralelas-igualdad de figuras por coincidencia o por } \\
\text { cantidad de magnitud. Esta opción epistemológica implicó que en el } \\
\text { diseño de la actividad de aula se comprometiera el uso de figuras y } \\
\text { el trabajo sobre el ángulo recto y las relaciones de paralelismo. }\end{array}$ \\
\hline $\begin{array}{l}\text { Desde lo } \\
\text { cognitivo }\end{array}$ & $\begin{array}{l}\text { Se identificaron tres dimensiones con respecto al sentido: la elabo- } \\
\text { ración de contenido, la identificación de valores y la determi- } \\
\text { nación de estatus. El diseño asumió la de elaboración, por ello } \\
\text { privilegió los procesos inferenciales abductivos e inductivos; el regis- } \\
\text { tro semiótico figural; el campo semántico geométrico euclidiano; la } \\
\text { organización discursiva argumentativa; y el proceso didáctico de re- } \\
\text { solución de problemas para establecer relaciones. }\end{array}$ \\
\hline $\begin{array}{l}\text { Desde la } \\
\text { organización } \\
\text { de las } \\
\text { interacciones } \\
\text { o el aspecto } \\
\text { socio-cultural } \\
\text { del aula }\end{array}$ & $\begin{array}{l}\text { La relación entre estudiante - estudiante y profesor - estudiante está } \\
\text { mediada por la estructura de la tarea: solución individual, solución de } \\
\text { parejas, solución colectiva e institucionalización. Eśto determina los } \\
\text { siguientes aspectos: el rol del estudiante en situación de elaboración } \\
\text { de sentido con respecto al contenido (no existe la restricción una única } \\
\text { respuesta). El ro/del profesor en situación de elaboración de sentido } \\
\text { desde el punto de vista didáctico. Así, la elaboración de sentido entre } \\
\text { estudiantes y profesor, con respecto al contenido es de tipo colec- } \\
\text { tivo. Un contrato didáctico fundamentado en la conciencia de pro- } \\
\text { ducción colectiva de conocimiento (implica la interacción con cono- } \\
\text { cimiento ya elaborado) y una actitud heurística colectiva con los } \\
\text { procesos de solución de problemas. }\end{array}$ \\
\hline
\end{tabular}

La propuesta realizada como diseño didáctico se caracterizó por ser un factor de desarrollo de un saber previo sobre la relación pitagórica. Por ello la pregunta fundamental planteada en los talleres, indagaba por relaciones matemáticas entre las que se requiere considerar esta relación. De ahí la exigencia de que esta relación haga parte del conocimiento elaborado anteriormente por quien resuelve el taller. 
Como criterios orientadores para el diseño de un momento de aprendizaje, establecimos: a) La necesidad de objetivar un saberya construido para comprenderlo, analizarlo, usarlo y darle un status epistémico teórico (entrar en una actividad metadiscursiva), mediante las enunciaciones realizadas. b) La necesidad de consolidar el saber, como garantía del aprendizaje. La experiencia de tratamiento de un conocimiento pone al sujeto, entre otras cosas, en la situación de establecer relaciones entre el objeto de conocimiento y los registros que lo representan (incluyendo el de la lengua natural); asimismo, las distintas representaciones de un objeto, posibilitan un mayor y más amplio conocimiento de ese objeto. Además, el paso a la verbalización de las elaboraciones que se realizan, en diferentes formas y con distintas funciones (narrar, explicar, argumentar), garantiza un desarrollo más consciente del conocimiento. c) La necesidad de configurar la situación de comunicación previa, con miras a asegurar el espacio de la construcción de sentidos.

Como factores que conformaron la situación de comunicación para el desarrollo de la relación pitagórica, establecidos por el taller, se identificaron tres: el contenido, la intencionalidady la estructura de la interacción. El diseño de los talleres propuso tres tipos de contenidos para la interacción: el referido a las orientaciones sobre las actividades que pide realizar (los de carácter instruccional); el referido a la construcción de pautas de trabajo (tanto la enunciación las actividades que se han de realizar como la construcción consciente de las pautas sobre cómo realizar óptimamente tales actividades); el referido al tema de la interacción (el tema que se va a tratar en la situación, que para el caso se trata de una relación matemática por inferir y las figuras geométricas por analizar). Se exige la aplicación de los conocimientos previos sobre: relaciones en general, relaciones matemáticas; sobre clases de figuras geométricas, operaciones con figuras y relaciones entre figuras (Ver talleres).

La intencionalidad de la interacción es escolarizante e investigativa y se condensa en estos dos propósitos: mejorar el saber matemático previo y promover una acción matemática (analizar, operar, producir explicaciones, discutir) y generar puntos de vista que serán valorados posteriormente.

De igual manera, la estructura de la interacción comunicativa identifica los roles que exige el diseño y los tipos de interacción que propone para la búsqueda de respuestas en la situación, conformando un sistema que configura la forma de comunicación que se presume necesaria para el desarrollo del conocimiento geométrico puesto en juego. Los tipos de roles fundamentales para esta tarea son el de argumentadory el de auditorio. El diseño didáctico estableció como requerimiento comunicativo, a partir del análisis de la situación, la necesidad de generar un proceso de interacción orientado a la generación de situaciones argumentativas 4 . De ahí que la metodología para el trabajo en el aula se desarrollara en tres momentos básicos: YO-YO(individual), $Y O-$ TÚ (pareja) y NOSOTROS-ELLOS (plenaria), con el fin de asegurar, tanto la construcción y preparación de respuestas individuales (como garantía de la construcción del

4 Confirmando lo que se había establecido en los resultados de la investigación previa descritos en el informe IDEP-Colciencias (2000) y publicados en el libro de Calderón, Dora y León, Olga (2001) Requerimientos didácticos y competencias argumentativas en matemáticas. La noción de situación argumentativa puede verse en el Capitulo lil de la tesis doctoral de la autora. 
argumentador|, como los momentos de discusión. De acuerdo con el diseño de tareas, el paso a un nuevo taller implica nuevos momentos YO-YO, YO-TÚ y NOSOTROS-ELLOS. La identificación de estos ciclos de elaboración permite considerar, de cierta manera, las distintas elaboraciones de los estudiantes e identificar factores de bloqueo y de desbloqueo en el desarrollo de los procesos cognitivos - discursivos realizados durante la secuencia didáctica. También aporta elementos para considerar, de manera particular, el papel del diseño de las tareas en el desarrollo de los procesos de los estudiantes. En la siguiente tabla se describe el juego de roles identificado en cada etapa del desarrollo y el tiempo empleado en cada etapa.

\begin{tabular}{l} 
Descripción de los roles \\
\hline 1. Trabajo individual: MOMENTO YO-YO \\
Cada estudiante resuelve la tarea asignada formulando por escrito la relación y las razo- \\
nes que la justifican.
\end{tabular}

2. Trabajo en parejas: MOMENTO YO-TÚ

Un miembro de la pareja asume el rol de argumentador y el otro miembro se constituye

- en auditorio.

La micro secuencia sugerida para esta interacción es:

a) El argumentador expone su solución con los argumentos que la soportan como una buena solución para la actividad.

b) El auditorio escucha, una vez ha concluido el argumentador, procede a realizar preguntas para aclarar aspectos sobre la presentación que ha hecho el argumentador.

c) El argumentador aclara las dudas del auditorio.

d) Si el auditorio se declara conforme con las aclaraciones, entonces se procede al paso siguiente, si no es así se vuelve a iniciar la ronda de aclaraciones.

e) El auditorio toma posición: se adhiere y completa la solución dada por el argumentador, o bien refuta al argumentador hasta convencerle de las fallas en sus procesos. En este momento, si el auditorio tiene otra solución, toma el rol de argumentador. para iniciar el ciclo nuevamente.

El proceso termina cuando se manifiesta la adhesión y se procede a elaborar, en conjunto, la solución para presentar al resto de sus compañeros en la plenaria.

3. Plenaria- debate de soluciones: MOMENTO NOSOTROS-ELLOS Una pareja asume el rol de argumentadora y el resto de parejas asumen el rol de auditorio. El proceso que se sigue es el mismo que el de parejas.

4. Institucionalización de lo elaborado: MOMENTO ÉL-NOSOTROS El profesor hace explícitas la solución elaborada y las razones que la justifican, según el proceso desarrollado colectivamente. Presenta una estructura del tipo de relaciones consideradas y del tipo de justificación propuesta. Orienta la valoración que se ha desarrollado durante el proceso en sus diferentes aspectos.

Para la regulación y el mantenimiento de una situación argumentativa en el aula, se identifican dos roles para el profesor: en la dimensión didáctica el de director para regular interacciones; centrar tópicos de reflexión, preguntar para potenciar procesos de solución, reorientar discusiones e institucionalizar elaboraciones parciales e identificar bloqueos. En la dimensión argumentativa, asumir, eventualmente, el rol de contraargumentador para obligar posiciones discursivas y epistémicas más fuertes en los 
estudiantes o para interpretar (como la conciencia del grupo) aspectos no satisfactorios en las soluciones defendidas por los estudiantes. De esta manera potencia la modelización de interacciones argumentativas en los estudiantes. Así pues, el estudio de las variables macroestructurales consideradas permitió explicitar una estructura didáctica conformada para favorecer una interacción argumentativa y un desplazamiento de procesos argumentativos no matemáticos a procesos argumentativos matemáticos.

El análisis a priori en su nivel microestructural permitió diseñar la secuencia de actividades definitiva, de acuerdo con los propósitos investigativos y didácticos y con los aspectos metodológicos planteados anteriormente. Este segundo nivel corresponde al análisis de los aspectos relacionados con el sentido propuesto por la tarea y con las acciones cognitivas y discursivas exigidas por ella, mediante los enunciados que la configuran. Así, la tarea se considera como una situación enunciativa que involucra al taller como enunciador y como enunciado y àl estudiante como enunciatariö. En esta situación, se toma como objeto de análisis la expansión discursiva (Martínez, 2001; Düval, 1999) manifiesta en los talleres propuestos (seis) y como categoría central el enunciadoy sus elementos constitutivos (proposición e intención) ${ }^{5}$. La siguiente tabla presenta una síntesis de esta secuencia y enseguida el resultado de su análisis microestructural.

ESTRUCTURA GENERAL DE LA SECUENCIA DE LOS TALLERES

\begin{tabular}{|c|c|c|c|c|}
\hline $\begin{array}{l}\text { OBJETIVO DE } \\
\text { APRENDI-ZAJE }\end{array}$ & $\begin{array}{l}\text { TRABAJO INDI- } \\
\text { VIDUAL }\end{array}$ & $\begin{array}{l}\text { TRABANO } \\
\text { DE PAREJA }\end{array}$ & PLENARIA & $\begin{array}{l}\text { F I G URAS } \\
\text { PROPUESTAS }\end{array}$ \\
\hline $\begin{array}{l}\text { Eormular } \\
\text { una relación } \\
\text { matemática } \\
\text { a partir del } \\
\text { análisis de } \\
\text { figuras. }\end{array}$ & $\begin{array}{l}\text { 1) Estudie el gráfico } \\
\text { presentado con } \\
\text { este taller y for- } \\
\text { mule la relación } \\
\text { matemática que } \\
\text { se muestra con el } \\
\text { gráfico. } \\
\text { 2) Eormule por lo } \\
\text { menos tres razo- } \\
\text { nes que le hacen } \\
\text { a usted concluir } \\
\text { esa relación. } \\
\quad-.\end{array}$ & $\begin{array}{l}\text { 1) Elaboraruna sola relación ma- } \\
\text { temática a partir de las rela- } \\
\text { ciones formuladas por los dos } \\
\text { integrantes. } \\
\text { 2) Proporcionar por lo menos } \\
\text { tres razones que le hacen a la } \\
\text { pareja concluir que esa es la } \\
\text { relación mostrada. } \\
\text { 3) Preparar una presentación } \\
\text { para convencer a las demás } \\
\text { parejas de que la relación. } \\
\text { elaborada por la pareja es la } \\
\text { más adecuada y de que las } \\
\text { razones que las soportan son } \\
\text { las más acertadas. A partir } \\
\text { de la presentación de una de } \\
\text { las parejas, elaborar la rela- } \\
\text { ción matemática más ade- } \\
\text { cuada para la gráfica propor- } \\
\text { cionada. }\end{array}$ & $\begin{array}{l}\text { A partir de la } \\
\text { presentación } \\
\text { de una de las } \\
\text { parejas, ela- } \\
\text { borar la rela- } \\
\text { ción mate- } \\
\text { mática más } \\
\text { adecuada } \\
\text { para la gráfi- } \\
\text { ca proporcio- } \\
\text { nada. }\end{array}$ & \\
\hline
\end{tabular}

5 El análisis a priori de las figuras que hacen parte de los talleres es presentado en la tesis de Olga Lucía León, citada anteriormente. Vale la pena señalar que, para efectos de la diferenciación de los campos dél lenguaje y de las matemáticas, en el análisis microestructural se especializan los dos tipos de análisis correspondientes con la didáctica de cada campo. Por esta razón, los dos análisis completos constituyen la totalidad del análisis a priori de las tareas. 
El análisis de la expansión discursiva atiende fundamentalmente al estudio del sentido y de la interacción propuestos en cada taller, con el fin de prever, desde esta propuesta enunciativa, las exigencias en el plano de la comprensión y en el de la interacción, que se le plantean al estudiante. De ahí que las variables analizadas en la expansión discursiva sean las operaciones heurísticamente pertinentes tanto para la elaboración del significado como para el desarrollo de la interacción. Tales operaciones, de tipo discursivo, son:

- De orden semiótico, dan razón del uso de registros como el de la lengua natural en relación con los demás registros, como el de las figuras. En este caso, se estudia la relación que aparece entre los registros de representación presentes en el texto y las que se prevé han de utilizar los estudiantes para resolver el problema.

- De orden semántico, que dan razón de la composición del significado y de la estructuración proposicional. Se estudian aquí las operaciones que se requieren para la comprensión de los significados expresados en los enunciados y para la reacción semánticamente pertinente a tales enunciados.

- De orden pragmático, que dan razón de la realización de la interacción intersubjetiva manifiesta en los enunciados. Se identifica, en este caso, el tipo de enunciado presentado en el taller, de acuerdo con la intencionalidad y la función que cumple cada uno de ellos, en relación con el interlocutor; además, se prevé el tipo de posicionamiento discursivo requerido por el enunciado.

Así pues, con base en la consideración de que el análisis de la expansión discursiva manifiesta en los talleres permite prever las acciones discursivas heurísticamente pertinentes, es posible determinar a priori los distintos aspectos para evaluar la efectividad de la tarea según los fines didácticos previstos e identificar obstáculos epistemológicos, cognitivos y discursivos que emergen en el aprendizaje. Además, el resultado del análisis a priori se convierte en el referente para la realización del análisis a posteriori. A continuación se presenta un ejemplo del resultado del análisis de la expansión discursiva diseñada para la tarea, teniendo como base los enunciados de los tres talleres básicos. De esta manera queda conformada la base para el análisis de los datos obtenidos con la aplicación de los talleres a la población estudiada.

\subsection{Conclusiones generales del análisis a priori de la expansión discursiva en los talleres}

Desde el punto de vista discursivo, este tipo de diseño propone una situación enunciativa orientada al desencadenamiento de una experiencia argumentativa en geometría. EI análisis de los talleres permite identificar la tarea como un enunciado macro que exige la puesta en marcha de operaciones de tipo discursivo para la comprensión y para la acción responsiva de parte del enunciatario-lector. Se reconoce aquí que la construcción del sentido de la propuesta instruccional diseñada para el estudiante, implica varios tipos de operaciones que ponen en juego las dimensiones cognitiva y comunicativa en la interacción escolar. En este proceso, las operaciones semánticas y semióticas posibilitan la relación que el sujeto-lector emprende tanto con el conocimiento en cues- 


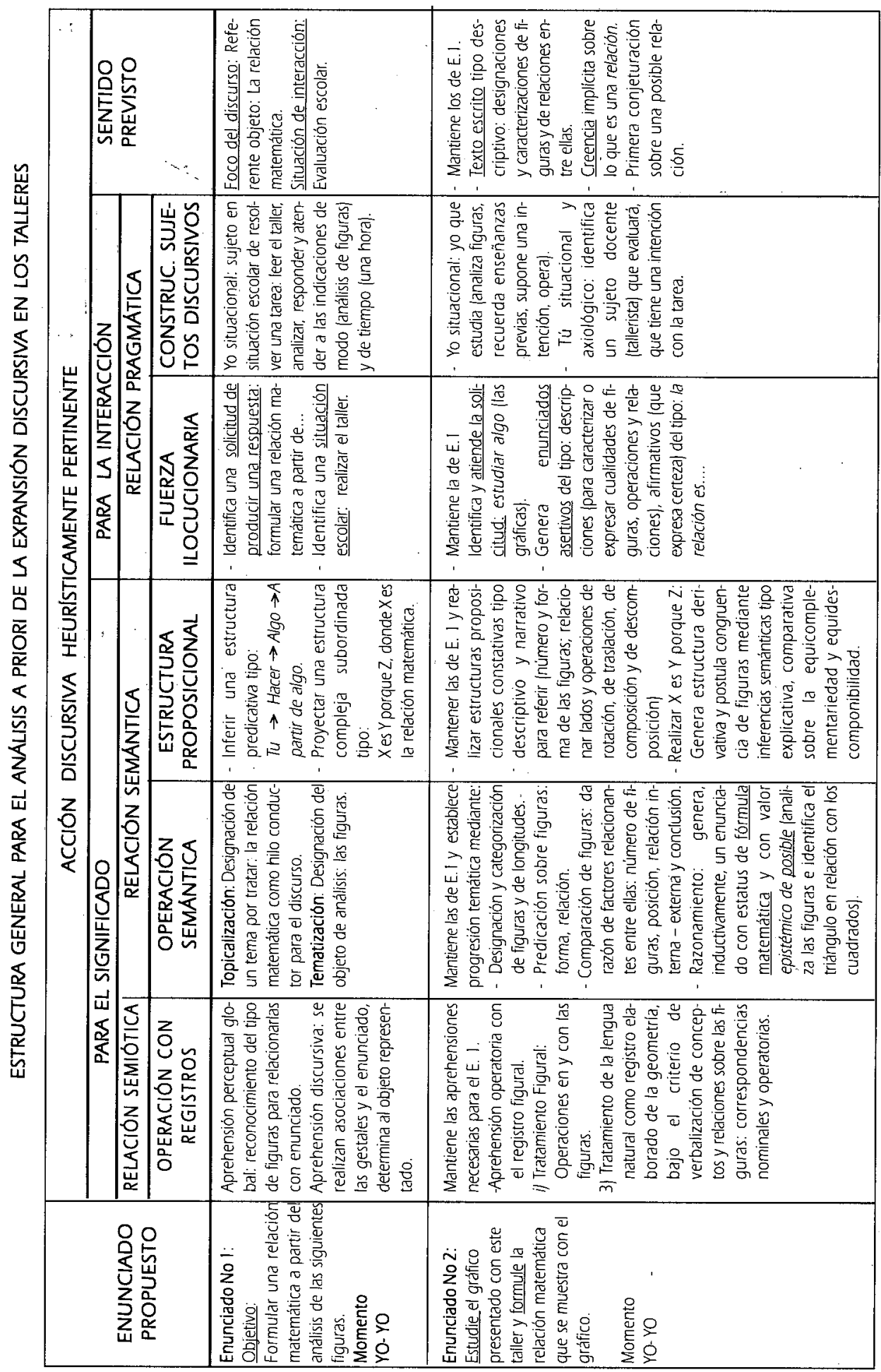


tión como con los interlocutores. Así, como macro enunciado, la tarea privilegia el uso de operaciones para:

- El tratamiento del contenido referido (la relación pitagórica y las figuras geométricas). Es decir, dado el carácter instruccional de los textos-talleres, el estudiante requiere poner en juego estrategias que le permitan operar con el conocimiento previo (sobre la geometría y sobre lo que es una relación matemátical y proyectar lá organización semántica y textual de ese conocimiento en relación con una exigencia de acción cognitiva y discursiva, que tiene que ver con la formulación de una relación. Se requiere, entonces, la comprensión sọbre lo pedido y la puesta en marćcha de lo exigido, cuando lo visible para el estudiante son las figuras geométricas própuestäs; así que hay cierta complejidad en el mantenimiento del tópico relación como tópico de la tarea. Este tipo de tratamiento, más analítico que descriptivo, pone al sujéto en situación de realizar razonamientos permanentemente; es decir, de realizar procesos metadiscursivos, que no son muy frecuentes en las actividades escolares comunes, e interacciones de tipo argumentativo, que exigen posicionamientos epistémicos y discursivos definidos y conscientes.

- La construcción de los interlocutores (compañero, auditorio, profesor) en relación con el contenido y con la situación. Esto quiere decir que la tarea exige el posicionamiento epistémico y discursivo del tallerista; una conciencia de lo que sabe y de to que comprende para relacionarse con sus interlocutores, de acuerdo con lo pedido por el taller (analizar, escuchar, discutir, consensuar) que es producir un sentido (una relación matemática). Complementariamente, como se observa en el análisis, la estrategia del taller de solución de problemas organizó tanto las formas de interacción con el conocimiento como las formas de comunicación de dicho conocimiento.

Vale la pena destacar que la comprensión de las exigencias cognitivas y discursivas presentes en la tarea revelan un nivel de complejidad de la tarea. Esto en tanto que se pretendió situar a los estudiantes en una experiencia de heuresis ${ }^{6}$ que permita la objetivación de los saberes matemáticos adquiridos previamente, con respecto a la relación pitagórica y poner al servicio de este proceso, la forma de interaćción y de producción discursiva. A continuación se presenta una muestra de los resultados de la aplicación de los talleres y el análisis a posterior realizado.

\footnotetext{
Aunque en los diccionarios comunes no se encuentra la palabra heuresis, se ha querido usar aquí para designar procesos altamente interpretativos que devienen de la propuesta textual. Es decir, aunque todo texto exige al lector realizar procesos de interpretación, aquellos que proponen explícitamente realizar análisis de sí mismos, en especial el análisis y la inferencia a partir de sus contenidos, plantearían al lector una relación más heurística que otros. El término heurístico hacé alusión al arte de interpretar o investigar fuentes históricas.
} 


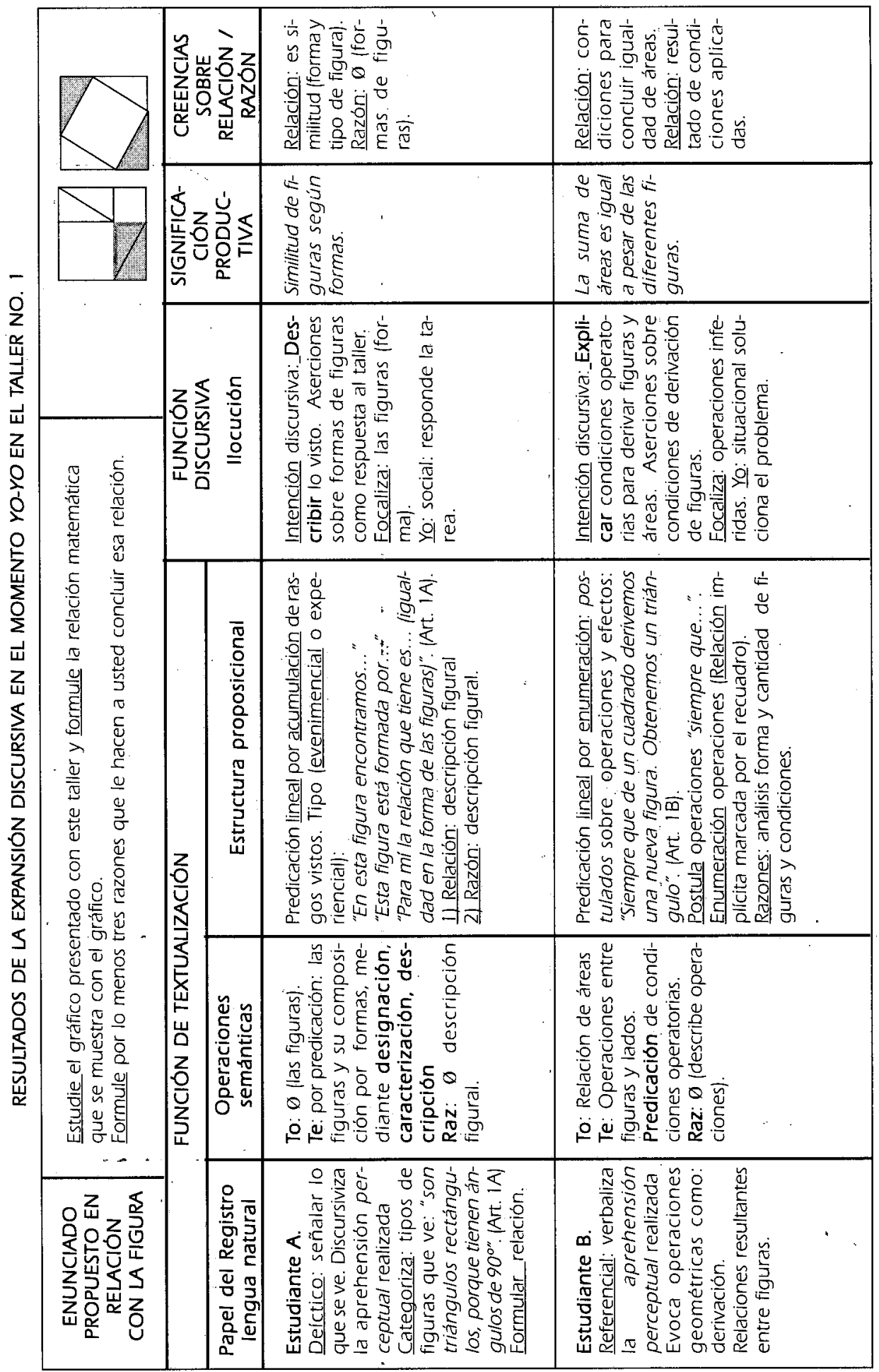




\begin{tabular}{|c|c|c|}
\hline 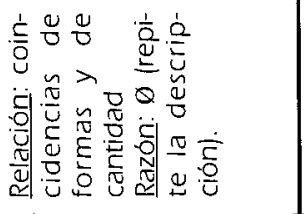 & 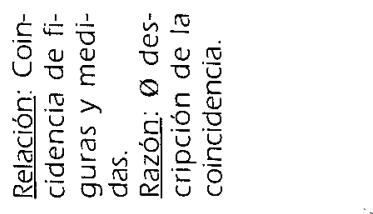 & 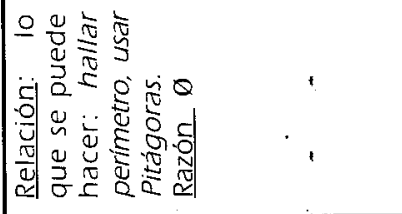 \\
\hline 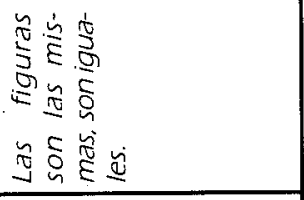 & 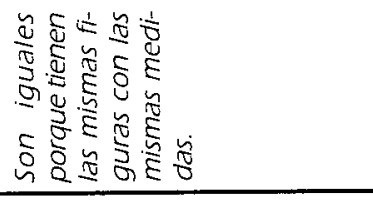 & $\begin{array}{l}0 \\
0 \\
0\end{array}$ \\
\hline 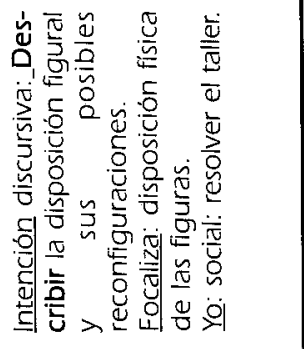 & 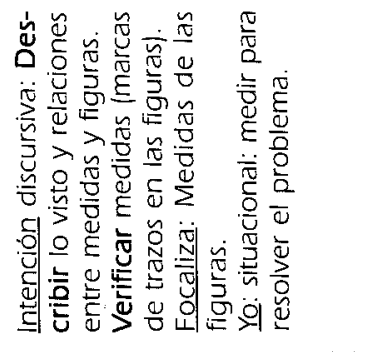 & 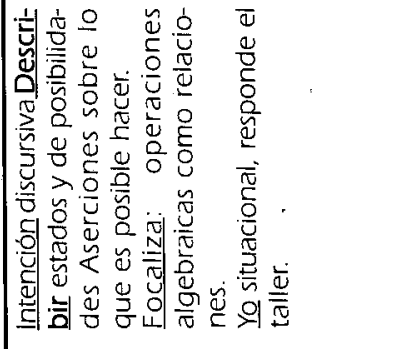 \\
\hline 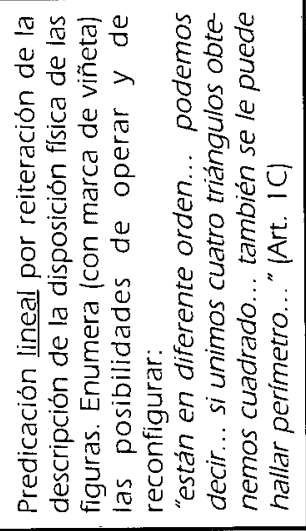 & 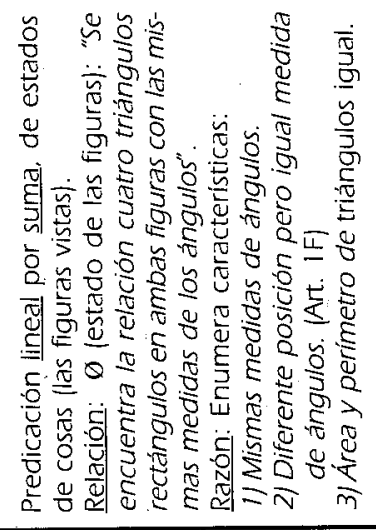 & 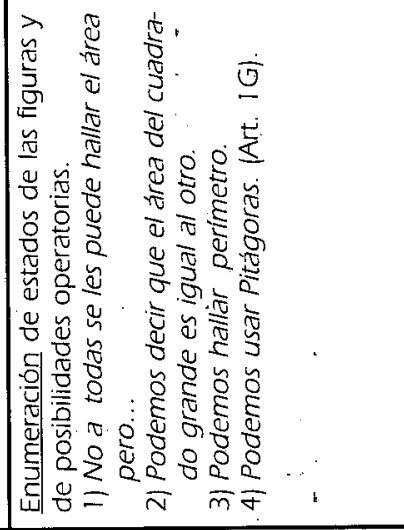 \\
\hline 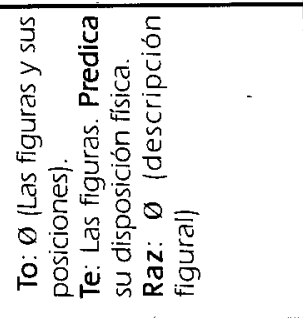 & 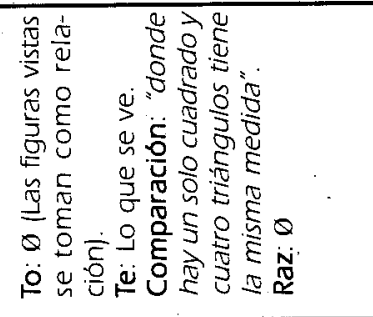 & 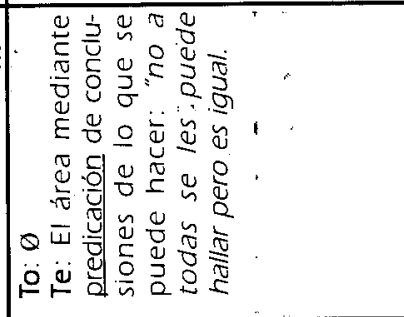 \\
\hline 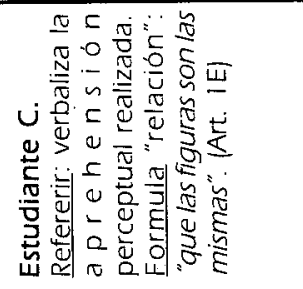 & 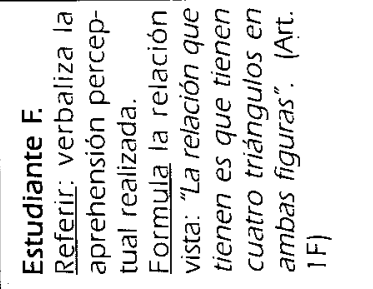 & 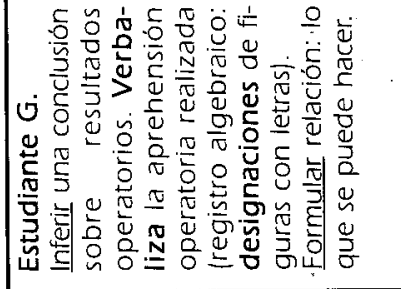 \\
\hline
\end{tabular}




\subsection{Conclusiones de.los resultados del momento YO-YO, Taller No. 1}

Todos los estudiantes realizan aprehensión discursiva, en general para verbalizar las otras aprehensiones de las figuras que han realizado. Entre las demás aprehensiones primó la de tipo perceptual. El registro figural se convirtió en el que potenció la actividad heurística de los estudiantes, por cuanto se tomaron las figuras como el objeto de análisis y actuó sobre este registro: La lengua natural, como registro de representación, cumplió una función de auxiliar del registro figural, en tanto que se empleó esencialmente para verbalizar las aprehensiones y las conclusiones realizadas a partir de las figuras, pero no como factor central en los procesos heurísticos. Se identifican dos funciones para la lengua natural como registro, en relación con el registro figural: la referencial y la apofántica y se realizan mediante operaciones como: la designación: o la deixis para señalar las figuras (designarlas con letras o con nombres); la categorización para identificar tamaño y forma; la descripción para establecer número de lados y disposición espacial de figuras; para referir las operaciones realizadas, a propósito de las figuras; la predicación describiendo o narrando a partir de la aprehensión realizada a las figuras; se producen enunciados que hablan de los aspectos anteriormente señalados.

Ningún estudiante realiza razonamientos sobre los enunciados que producen para asignar valor o estatus a tales enunciados. Privilegian la descripción figural o la inferencia abductiva de operaciones posibles para verificar igualdad de área, de acuerdo con el conocimiento previo que tienen acerca de cómo se procede en matemáticas; también se producen, en algunos momentos, inferencias de tipo deductivo e inductivo para concluir operaciones posibles.

Los resultados muestran que la mayoría de los estudiantes NO topicalizan explícitamente una relación matemática: quienes lo hacen implícitamente no es sobre la esperada o tan sólo enuncian el término relación sin tomarlo después como tópico discursivo. En general se establece como tópico discursivo las figuras y sus características y también se tematiza sobre las figuras: medidas, formas, cantidad. Un estudiante da indicios de topicalizar una relación matemática, dado que reconoce que le están pidiendo hallar una relación matemática. Desde este punto de vista, lo que se genera en esta primera etapa no es un desarrollo de un sentido de relación matemática, sino una descripción de percepciones e inferencias acerca de las figuras.

Los estudiantes generan estructuras proposicionales lineales para describir figuras y características u operaciones. En aigunos casos se presentan estructuras evenimenciales (donde se enuncia la experiencia de ver); en este caso, la función agente la desempeña el estudiante y lo predicado es el objeto visto: las figuras. Se observa que el requerimiento pertinente de "producir estructuras proposicionales complejas con función explicativa" que evidenciara el acto de predicar sobre la categoría "relación" NO se manifestó explícitamente en este momento. En la mayoría de los casos los estudiantes enuncian con la intención de describir lo visto o lo inferido; uno de ellos también expresa intención verificativa. Prima la intención de describir con el fin de "explicar", hacerse entender $y$, por ello predominan actos de habla asertivos para informar; desde este punto de vista se observa una orientación discursiva más intencional en relación con la necesidad de satisfacer el requerimiento de la tarea, que en relación con la solución del problema. De ahí que prime la focalización de las formas figurales. En este sentido, no se realizó, en este primer momento la focalización de una relación matemá- 
tica, determinando una elaboración de significación dependiente del contexto más inmediato (las figuras).

Se manifiesta un privilegio del yo social, que pretende contestar el taller, posicionándose como el estudiante que participa en un proceso, que coopera. También se manifiesta un yo situacional que expresa un mayor interés por el uso de recursos para encontrar una respuesta al reto que pone la tarea, manifestándose en ellos una orientación hacia el objeto y un posicionamiento del sujeto que se cuestiona. No se presenta una evidencia de la existencia de certezas o dudas sobre "la relación" formulada, aunque sí una manifestación del yo que realizó una actividad. En este momento se privilegia un posicionamiento discursivo más social en relación con la respuesta al taller. '

Desde el punto de vista de la producción de significación, prima una significación de igualdad de figuras. Se observa una relación entre significado construido y tipo de aprehensión figural: se privilegió una aprehensión perceptual. Se evidencia también, en la expresión de significados, la manifestación de relaciones matemáticas, aunque los estudiantes no las tomen como tales y no construyan sobre ellas el tópico discursivo. Complementariamente, una concepción predominante que emerge sobre la categoría relación es la manifestada en términos de lo que coincide -tanto en forma como en cantidad- La categoría razón se asimila con predicado o descripción de la relación. Un estudiante presenta significados distintos para la categoría relación: operación y resultado. Se puede inferir, entonces que la emergencia de las concepciones se da por una relación ligada a la inmediatez de "lo visto": la forma y la cantidad en las figuras.

La descripción anterior establece la primera relación semántica y discursiva que se produce frente a la relación pitagórica (RP). A continuación se presentan conclusiones generales del estudio.

\section{CONCLUSIONES}

La articulación que se produjo entre los factores asociados a cada una de las tres variables que se consolidaron como constitutivas del género pedagógico ratificó la noción de género discursivo pedagógico como escenario sociocultural de un tipo de prácticas sociales, en este caso las prácticas pedagógicas. Pero más allá de esta ratificación, los resultados obtenidos también permitieron identificar particularidades del género discursivo pedagógico que emergieron durante el desarrollo del diseño didáctico; tales resultados nos llevan a considerar la postulación de un subgénero del gran género pedágógico: éste es el subgénero didáctico (Calderón y León, 2005). De ahí surge la necesidad de considerar la categoría situación didáctica ${ }^{7}$ como situación de comunicación en el aula, que se realiza bajo un contrato didáctico (Venturini, 2002); como un dispositivo que surge del análisis y de la puesta en relación de lôs elementos que conforman la triada

En este momento vale la pena señalar que "situación didáctica" se toma desde la perspectiva de género discursivo, como contexto de comunicación. No se trata, para este caso, de la noción de situación didáctica restringida a la teoría de las situaciones didácticas de Brousseau, en donde se entiende Ia S.D. como un esquema de modelización para un aprendizaje en particular, que incorpora el sistema del estudiante y el sistema del conocimiento y el sistema de problemas que los ponen en relación. 
didáctica y que se ponen en juego en la acción didáctica: el conocimiento escolar que se quiere movilizar; los estudiantes, sus saberes previos y los procesos que se pretenden desarrollar; y el profesor, su episteme y su propuesta de su diseño didáctico (tanto para la relación de los estudiantes con el saber como para la interacción).

El docente podrá establecer una relación más clara con el diseño didáctico (D.D.). Las exigencias de tipo epistemológico (disciplinar e interdisciplinar) y metodológico que impone la construcción de un D.D., como ya se observó en ese estudio, evidencian la necesidad de que el profesor cuente con un referente disciplinar y curricular que ejerza la vigilancia epistemológica de su acción didáctica. Un ejemplo de esta relación la constituye el ejercicio realizado en este estudio con el diseño de la secuencia didáctica y análisis a priori de las tareas. He ahí un reto para la formación de docentes.

El taller, como propuesta metodológica, para el trabajo en el aula reiteró su naturaleza de ser un espacio para la aplicación de saberes elaborados previamente. Es decir, se evidenció que la realización de un taller Sí exige unas condiciones epistémicas y discursivas mínimas que permitan a los talleristas movilizar las relaciones y aplicaciones de conocimientos que sus propósitos exijan. Este hecho se evidenció en la dificultad de formular la RP y las razones que la sustentaran: faltaba elaboración previa de saberes relacionados con la $R P$.

El reto cognitivo (desde el punto de vista matemático) y discursivo (desde el punto de vista de la comunicación de lo matemáticol de formular relacionesy de buscarrazones exigió un tratamiento particular de la información proveniente de los talleres y de la que se tenía previamente. Se requería pasar de la aprehensión puramente perceptual (que era necesaria) a la operatoria y a la discursiva y de la expansión discursiva que refiere y describe "lo visto", que depende del contexto inmediato, a la expansión discursiva que explica y justifica, que se desprende discursivamente de ese contexto inmediato. Es decir, la tarea se planteó como una actividad prioritariamente heurística en la que formular relaciones y establecer razones implicaba hacer una entrada a las teorías y exigía destrezas en el tipo de registro presentado (el figural). Esta era la condición epistémica de la que no disponían los estudiantes. Por esta razón, la formulación de la RP. tuvo que esperar, hasta que ellos lograron un mínimo de comprensiones y de certezas sobre los saberes asociados a la RP; mientras tanto, las significaciones producidas se mostraron poco elaboradas discursivamente y bastante restringidas al contexto inmediato, el de las figuras vistas en los talleres.

\section{BIBLIOGRAFÍA}

ARTIGUE, M., DOUADY, R, Gómez, P. (Ed). (1995). Ingeniería didáctica en educación matemática, "una empresa docente". México: Grupo Editorial Iberoamérica. Págs. 33-59.

BLOCH, I., (2002). Differents niveaux de modéles de milieu dans la théorie des situations en: Dorier, J., Artaud., Artigue, M., Berthelot, R., Floris, R., (Eds). Actes de la 11 Ecole D'Ete de Didactique des Mathematiques. Paris: La pensée Sauvage Editions. 
BROUSSEAU, G.(2004) "Fondements et méthodes de la didactique". En Théorie des situations didactiques. Grenoble: La Pensée Sauvage, Editions.

CALDERÓN, Dora Inés y LEÓN, Olga Lucía (2001) Requerimientos didácticos y competencias argumentativas en matemáticas. Bogotá: IDEP - Colciencias.

CHEVALLARD, Y (1982). Sur l'ingénierie didactique. Texte prépare pour la douxieme Ecole D'ete de Didactique des Mathematiques. Orleáns Juillet 1982.

DUVAL, Raymond. (1999). Semiosis y pensamiento humano: Registros semióticos y aprendizajes intelectuales. (Traducción de Miryam Vega). Cali: Universidad del Valle.

LEÓN, OIga Lucía y CALDERÓN, Dora Inés. (2003). Argumentar y validar en matemáticas. ¿Una relación necesaria?. Bogotá: Universidad del Valle- Colciencias.

MARTÍNEZ, María Cristina (1997) Análisis del discurso. Cali: Universidad del Valle. 164 págs.

MARTíneZ, María Cristina. (2004b). "La construcción de los sujetos discursivos, la orientación social de la argumentación en la dinámica enunciativa del discurso". Conferencia presentada en el 3er Coloquio Nacional de Estudios del Discurso. Medellín, septiembre 22 a 24 de2004.

. (2005). La construcción del proceso argumentativo en el discurso. Perspectivas teóricas y talleres. Cali: Cátedra UNESCO- Universidad del Valle. 299 Págs.

PERELMAN, Chäim y OLBRECHTS-TYTECA, Lucien. (1989). Tratado de la Argumentación. Madrid: Gredos. 